\title{
Strain tensors in layer systems by precision ion channeling measurements
}

H. Trinkaus, D. Buca, B. Holländer, R. A. Minamisawa, S. Mantl, and J. M. Hartmann

Citation: Journal of Applied Physics 107, 124906 (2010);

View online: https://doi.org/10.1063/1.3415530

View Table of Contents: http://aip.scitation.org/toc/jap/107/12

Published by the American Institute of Physics

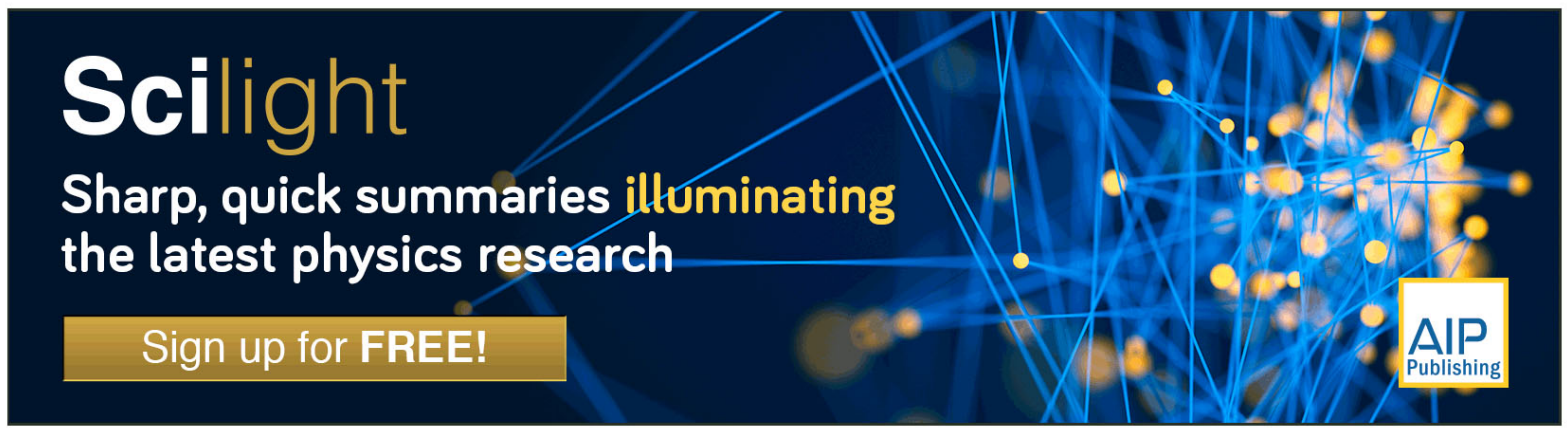




\title{
Strain tensors in layer systems by precision ion channeling measurements
}

\author{
H. Trinkaus, ${ }^{1}$ D. Buca, ${ }^{1, a}{ }^{1,}$ B. Holländer, ${ }^{1}$ R. A. Minamisawa, ${ }^{1}$ S. Mantl, ${ }^{1}$ and \\ J. M. Hartmann ${ }^{2}$ \\ ${ }^{1}$ Institute of Bio- and Nanosystems (IBN 1) and JARA-Fundamentals of Future Information Technology, \\ Forschungszentrum Juelich, D-52425 Juelich, Germany \\ ${ }^{2}$ CEA-LETI, MINATEC, 17 Rue des Martyrs, F-38054 Grenoble, Cedex 9, France
}

(Received 27 November 2009; accepted 29 March 2010; published online 24 June 2010)

\begin{abstract}
A powerful method for analyzing general strain states in layer systems is the measurement of changes in the ion channeling directions. We present a systematic derivation and compilation of the required relations between the strain induced angle changes and the components of the strain tensor for general crystalline layer systems of reduced symmetry compared to the basic (cubic) crystal. It is shown that, for the evaluation of channeling measurements, virtually all layers of interest may be described as being "pseudo-orthorhombic." The commonly assumed boundary conditions and the effects of surface misorientations on them are discussed. Asymmetric strain relaxation in layers of reduced symmetry is attributed to a restriction in the slip system of the dislocations inducing it. The results are applied to $\{110\} \mathrm{SiGe} / \mathrm{Si}$ layer systems. (C) 2010 American Institute of Physics. [doi:10.1063/1.3415530]
\end{abstract}

\section{INTRODUCTION}

High elastic strains and stresses can substantially modify physical properties of surface near layers. An important example is the effect of strain/stress on the electronic properties of semi-conducting surface layers. Misfit strains and stresses in SiGe layers grown on Si substrates, for instance, can reach up to a few percent corresponding to a few gigapascal. ${ }^{1}$ In fact, strain engineering has meanwhile become an important and necessary technique to improve advanced nanoelectronic devices. $^{2}$ Advanced methods have been proposed to relax strained SiGe layers ${ }^{3,4}$ and to transfer strain increments from a relaxing SiGe layer to a $\mathrm{Si}$ cap layer. ${ }^{5}$ The aim of such methods is to increase carrier mobilities ${ }^{6}$ and dopant solubilities $^{7}$ in Si layers.

Apart of various $\mathrm{x}$-ray ${ }^{8,9}$ and electron diffraction techniques, ${ }^{10}$ a powerful method for the nondestructive characterization of strain states in thin layer systems is Rutherford backscattering spectroscopy in the ion channeling mode (RBS/C). ${ }^{11,12}$ The applicability of this method is based on the well known fact that strains in crystals are generally associated with changes in the angles between different crystal directions - except for purely hydrostatic strains. The accuracy for deducing strain states from channeling directions is highest for low index directions, for instance $\langle 100\rangle,\langle 110\rangle$, and $\langle 111\rangle$ in cubic crystals. Limitations of the method are expected for ultrathin layers consisting only of a few atomic layers. 1,13

Holländer et al. ${ }^{14}$ have described a high-precision $\mathrm{RBS} / \mathrm{C}$ goniometer allowing rotations with an angular resolution of $0.005^{\circ}$. The associated error is so small that it does not significantly affect the accuracy of strain determination which is, in fact, determined by the physics of channeling. The simple examples shown in Fig. 1 indicate an angular accuracy in determining a certain channeling direction (rep-

${ }^{a)}$ Electronic mail: d.m.buca@fz-juelich.de. resented by the location of the dips in the channeling profile) and the strain induced changes in angles between two directions of about $0.03^{\circ}$ and $0.042^{\circ}$, respectively. This would correspond to a strain increment of about $0.075 \%$. The uncertainty in determining a certain component of the strain tensor is generally higher, around $0.1 \%$, depending on the number of angle changes required to deduce it as will be shown below.

In the past, mainly simple planar $\{100\} \mathrm{SiGe} / \mathrm{Si}$ layer systems characterized by plane stress and tetragonal strain states have been analyzed by RBS/C combined with a plane stress/ biaxial strain model (assuming that the real surface coincides with the ideal). ${ }^{1,3,5}$ Only recently, $\{100\} \mathrm{SiGe} / \mathrm{Si}$ layer systems structured in the form of $\mathrm{SiGe}$ stripes in a $\langle 110\rangle$ direction on Si substrates have been considered with the goal to generate, by asymmetric strain relaxation in the SiGe stripes, more favorable stress states close to the uniaxial limiting case in $\mathrm{Si}$ cap layers ${ }^{15}$ for which increased carrier mobility is expected. In this respect, other promising options are planar or patterned $\{110\}$ or $\{111\}$ layer systems. In fact, the choices concerning the type of layer planes and directions of stripes are virtually limited to $\{100\},\{110\}$, and $\{111\}$ planes and $\langle 110\rangle$ directions, respectively, due to limitations in sample preparation. In changing the type of the layer plane from $\{100\}$ to $\{110\}$ and $\{111\}$, the layer symmetry changes from tetragonal to orthorhombic and trigonal, respectively. In structuring $\{100\}$ and $\{111\}$ layer systems, the symmetry is (further) reduced to orthorhombic and monoclinic, respectively, (see Table I).

It is useful here to give the reader an idea of the types of layer systems which we consider to be analyzed by RBS/C. The specific layer within the system, the strain state of which is to be determined is assumed to be approximately chemically homogeneous and homogeneously strained, at least over the analysis depth of the $\mathrm{He}^{+}$-ions used in RBS/C (about $10 \mathrm{~nm}$ to $1 \mu \mathrm{m})$. It is monocrystalline and essentially planar over the scale of the bombarded area $(0.5$ to $5 \mathrm{~mm} \emptyset)$. It is 

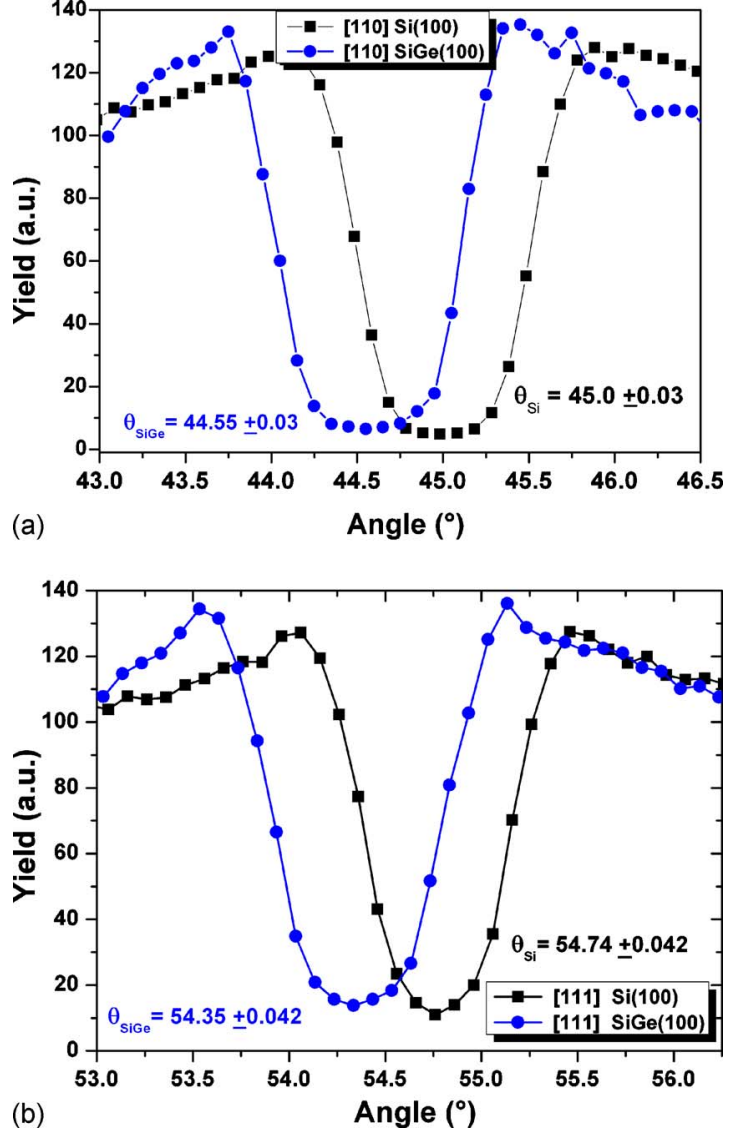

FIG. 1. (Color online) Examples for measured channeling angular yield scans, (a) around $a[101]$ and (b) around $a[111]$ direction through (010) and (110) plans of unstrained $180 \mathrm{~nm}$ thick (001)Si and pseudomorphic $\mathrm{Si}_{0.77} \mathrm{Ge}_{0.23}$, respectively. The angular accuracy in determining a channeling direction defined by the location of the minimum yield is estimated to be about $0.03^{\circ}$

connected (not necessarily epitaxially) with a substrate and may be covered by a thin layer. To obtain clearly separated backscattering signals it is advantageous if the considered layer contains heavier elements than the substrate, for instance, $\mathrm{SiGe}$ on $\mathrm{Si}$. If, for specific reasons, a cap layer is used, its thickness should be limited to values $<10 \mathrm{~nm}$ to avoid disturbing beam steering. The strain state of the layer is determined by its connection with the substrate, possibly present misfit dislocations in the interface and the surface boundary conditions (see below). Misfit dislocations are inevitably connected with intrinsic variations in the strain field. The strain is effectively homogeneous when the distance between the misfit dislocations is smaller than the thickness of the layer. A layer system as sketched here is shown for illustration in Fig. 2.

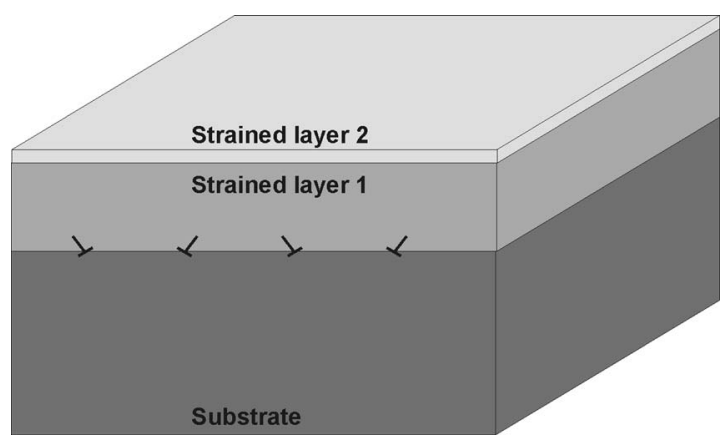

FIG. 2. Typical layer system envisaged in modern strain engineering. The strain in layer 1 is considered to be analyzed by RBS/C. Misfit-dislocations in the interface to the substrate inducing partial strain relaxation are indicated.

For deducing strains in layers from RBS/C measurements, the relations between changes in angles between crystal directions and strain tensor components are needed. Even though the derivation of such relations is straightforward it becomes increasingly more complicated with decreasing symmetry of the layer. In order to help possible users of the method, we present in this study a systematic derivation and compilation of complete sets of relations between changes of angles between suitably chosen crystal directions and all principally accessible components of the strain tensor for the most important layer systems in cubic crystals. We discuss the validity of the commonly assumed boundary conditions and their testing by RBS/C.

The present analysis has been motivated by a very recent examination of a pure uniaxiality of strain relaxation in $\{110\} \mathrm{SiGe} / \mathrm{Si}$ predicted on the basis of the dislocation dynamics assumed to control the relaxation process. In the publication of the results, ${ }^{16}$ the formulas required to evaluate the RBS/C measurements have been taken from the present work. In a final section, we will discuss the results presented in Ref. 16 as an application of our present analysis.

\section{STRAIN TENSORS BY CHANNELING}

\section{A. Relations between angle changes and strain}

It is well known that strains in crystals are generally associated with changes in the angles between different crystal directions-except for direction conserving purely hydrostatic strain, i.e., dilatation or compression. According to the latter restriction, only the "deviatory" part, $\widetilde{\boldsymbol{\varepsilon}}$, defined as the difference between the full strain tensor $\boldsymbol{\varepsilon}$ and its hydrostatic part, can be determined by channeling measurements, i.e.,

$$
\widetilde{\boldsymbol{\varepsilon}}=\boldsymbol{\varepsilon}-\operatorname{trace}(\boldsymbol{\varepsilon} / 3) \cdot \mathbf{1}=\left(\begin{array}{ccc}
\left(2 \varepsilon_{11}-\varepsilon_{22}-\varepsilon_{33}\right) / 3 & \varepsilon_{12} & \varepsilon_{13} \\
\varepsilon_{12} & \left(2 \varepsilon_{22}-\varepsilon_{11}-\varepsilon_{33}\right) / 3 & \varepsilon_{23} \\
\varepsilon_{13} & \varepsilon_{23} & \left(2 \varepsilon_{33}-\varepsilon_{11}-\varepsilon_{22}\right) / 3
\end{array}\right) \text {, }
$$


where $\mathbf{1}$ is the diagonal unit tensor. The trace of $\widetilde{\boldsymbol{\varepsilon}}$ vanishes by construction. Therefore the diagonal elements of $\widetilde{\varepsilon}$ are not linearly independent of each other, meaning that one of them can be expressed as a linear combination of the two others. The following analysis shows that the five independent elements of $\widetilde{\boldsymbol{\varepsilon}}$ can be determined by channeling measurements, irrespective of any boundary conditions.

The basis of our subsequent analyses is a general expression for the strain induced change of the angle $\theta \rightarrow \theta^{\prime}$ between two vectors $(\mathbf{P}, \mathbf{Q}) \rightarrow\left(\mathbf{P}^{\prime}, \mathbf{Q}^{\prime}\right)$ representing two crystal directions (channeling directions or normal vectors on channeling planes) as illustrated in Fig. 3. We assume that the distortion of the considered layer is effectively homogeneous over the range of the investigated area. Large scale rotations are assumed to be taken into account by proper adjustments of the RBS/C goniometer and may therefore be neglected without loss of generality, meaning that the displacement gradient tensor can be taken to be equal to the strain tensor $\boldsymbol{\varepsilon}$. In this case, the relation between two vectors in the strained lattice, $\mathbf{P}^{\prime}, \mathbf{Q}^{\prime}$, and the original lattice, $\mathbf{P}, \mathbf{Q}$, may be represented as

$$
\mathbf{P}^{\prime}=\mathbf{P}+\varepsilon \cdot \mathbf{P}, \mathbf{Q}^{\prime}=\mathbf{Q}+\varepsilon \cdot \mathbf{Q}
$$

The angle, $\theta^{\prime}$, between the two vectors in the strained lattice may be written, analogously as for the unstrained lattice, in the form

$$
\cos \left(\theta^{\prime}\right)=\left(\mathbf{P}^{\prime} \cdot \mathbf{Q}^{\prime}\right) /\left(\mathbf{P}^{\prime 2} \mathbf{Q}^{\prime 2}\right)^{1 / 2}
$$

Linear (up to first order) expansion of $\cos \left(\theta^{\prime}\right)$ with respect to the angle change $\delta=\theta^{\prime}-\theta$, and of the right hand side of Eq. (3) with respect to the strain tensor $\boldsymbol{\varepsilon}$, respectively, yields, in vector/tensor component representation,

$$
\begin{aligned}
\operatorname{tg}(\theta) \delta(\mathbf{P}, \mathbf{Q}, \boldsymbol{\varepsilon}) \approx & P_{i} \varepsilon_{i j} P_{j} / P_{k} P_{k}+Q_{i} \varepsilon_{i j} Q_{j} / Q_{k} Q_{k} \\
& -2 P_{i} \varepsilon_{i j} Q_{j} / P_{k} Q_{k}
\end{aligned}
$$

for $P_{k} Q_{k} \neq 0, \quad \theta \neq \pi / 2$, and

$$
\delta(\mathbf{P}, \mathbf{Q}, \boldsymbol{\varepsilon}) \approx-\left(2 P_{i} \varepsilon_{i j} Q_{j}\right) /(P Q),
$$

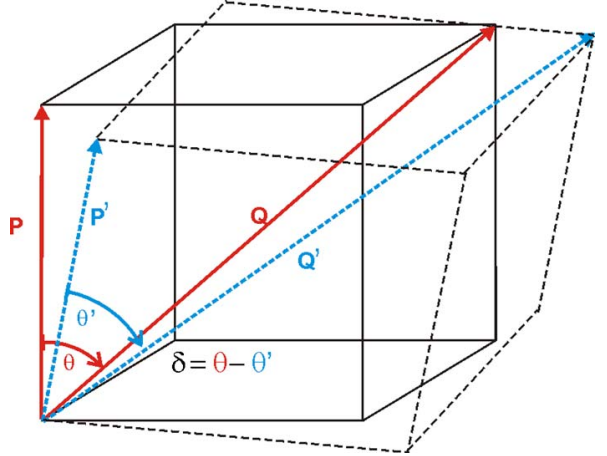

FIG. 3. (Color online) Illustration of a strain induced angle change $\theta \rightarrow \theta^{\prime}$ between two vectors $(\mathbf{P}, \mathbf{Q}) \rightarrow\left(\mathbf{P}^{\prime}, \mathbf{Q}^{\prime}\right)$ representing two crystal directions (lattice vector in "pole" directions and body diagonal).

for $P_{k} Q_{k}=0, \quad \theta=\pi / 2$,

where Einstein's convention of summing over repeated indices is to be used. According to Eqs. (4a) and (4b), the angle change vanishes, as expected, for purely hydrostatic strain, $\varepsilon_{i j}=\boldsymbol{\delta}_{i j}$ (where $\boldsymbol{\delta}_{i j}$ is the diagonal unit tensor in Kronecker's notation) confirming formally that channeling measurements can only provide the deviatory part of the strain tensor, $\widetilde{\boldsymbol{\varepsilon}}$. For strains of the order of $1 \%$, the relative error in the linear approximations given by Eqs. (4a) and (4b) is of the same order, i.e., about $1 \%$, which is much smaller than the error resulting from the experimental uncertainty in measuring angles.

Applying Eq. (4a) for given pole direction, $\mathbf{P}$, to different, appropriately chosen other (channeling) directions oblique to the pole, $\mathbf{Q}$, linearly independent equations for (certain) components of $\widetilde{\boldsymbol{\varepsilon}}$ can be derived which can then be solved for those components. For deriving the complete fivecomponent deviatory strain tensor from such angle changes, at least five linearly independent relations of the form of Eq. (4a) are needed, meaning that at least five different directions $\mathbf{Q}$ in at least three channeling planes containing the pole direction $\mathbf{P}$ must be examined. For cubic systems, appropriate sets of directions are illustrated in Fig. 4 and listed in Table I and II. For complete sets of the stereographical plots of crystal directions see Ref. 17.

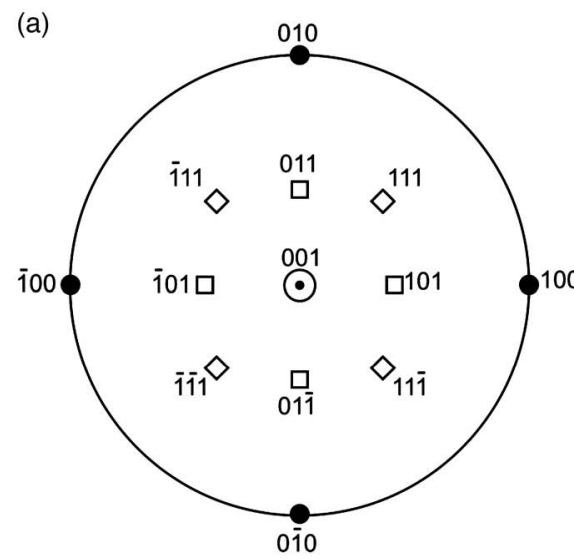

(b)

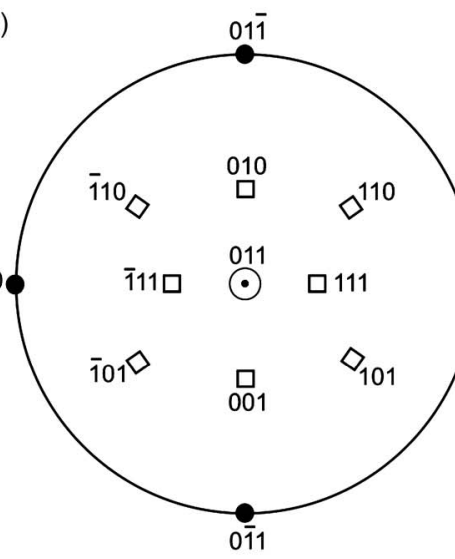

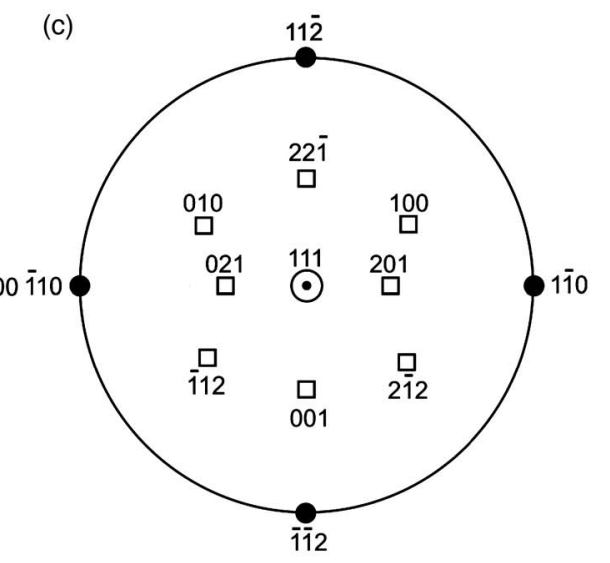

FIG. 4. Stereographic projections of crystal directions recommended for examination of (a) $\{100\}$, (b) $\{110\}$, and (c) $\{111\}$ layer systems on cubic crystals by $\mathrm{RBS} / \mathrm{C}$. 
For discussing the physics revealed by channeling measurements, it is useful to consider the strain tensor in a coordinate system adapted to the symmetry of the layer (distinguished in the following from the standard coordinate system of the crystal structure by apostrophes). One way to obtain the strain tensor in such a layer adapted system is to first derive it in the standard crystal system and to transform it then to the layer system. We have used this method for all layer types listed in Table I. Though straightforward, this procedure becomes increasingly tedious with progressing reduction in the layer symmetry.

For virtually all cases of interest (comprising the main layer orientations in orthorhombic, tetragonal, hexagonal, and, particularly, in cubic crystals as listed in Table I) it is, however, sufficient and simpler to evaluate Eq. (4) for an effectively orthorhombic system with lattice vectors $\mathbf{a}, \mathbf{b}, \mathbf{c}$ in the properly chosen base and pole axis directions 1,2, 3, of the original lattice, for instance, $\mathbf{a} \rightarrow[1 \overline{1} 0], \mathbf{b} \rightarrow[11 \overline{2}], \mathbf{c}$ $\rightarrow[111]$ for (111)-layers in cubic crystals considered in column 5 of Table I. Even though such layers have only trigonal/monoclinic symmetry, the distribution of their crystal directions, irrespective of their type, is "pseudoorthorhombic" as demonstrated by the stereographic projection shown in Fig. 4(c). For the strain induced change in the angle between the pole direction [001] and the direction [111] in an orthorhombic layer of lattice constants $a, b, c$, Eq. (4a) yields (apostrophes neglected for simplicity):

$$
\begin{aligned}
& \left(a^{2}+b^{2}+c^{2}\right)\left(\sqrt{a^{2}+b^{2}} / c\right) \delta_{111}=a^{2} \varepsilon_{11}+b^{2} \varepsilon_{22}+2 a b \varepsilon_{12} \\
& -\left(a^{2}+b^{2}\right)\left[\varepsilon_{33}+2\left(a \varepsilon_{13}+b \varepsilon_{23}\right) / c\right] .
\end{aligned}
$$

Since, for angles between directions, the reference direction is always the pole, the latter need not be included in the labeling of the angle change, here in $\delta_{111}$. Analogous expressions for other angle changes are obtained from Eq. (5) by properly choosing $a, b$, and $c \neq 0$. Directions in the pseudoorthorhombic layer system may easily be expressed in terms of directions in the underlying crystal lattice system by simply summing base and pole vectors properly, for instance $[101]^{\prime}=[1 \overline{1} 0]+[111]=[201]$ for the (111)-layer system.

Examination of changes in angles between the pole direction and two pairs of directions with projections parallel to the two base axes, for instance, allows to determine the two diagonal elements, $\varepsilon_{11,22}^{\prime}-\varepsilon_{33}^{\prime}$, and the two off-diagonal shear elements, $\varepsilon_{13}^{\prime}, \quad \varepsilon_{23}^{\prime}$ in the orthorhombic coordinate system. Choosing the low index directions $[101]^{\prime}$ and [101]', we find:

$$
\begin{aligned}
& 2\left(\varepsilon_{11}^{\prime}-\varepsilon_{33}^{\prime}\right) \approx(a / c+c / a)\left\{\delta_{101}^{\prime}+\delta_{101}^{\prime}\right\}, \\
& 4 \varepsilon_{13}^{\prime} \approx-\left(1+c^{2} / a^{2}\right)\left\{\delta_{101}^{\prime}-\delta_{101}^{\prime}\right\},
\end{aligned}
$$

The results for $\varepsilon_{22}^{\prime}-\varepsilon_{33}^{\prime}$ and $\varepsilon_{23}^{\prime}$ follow from Eqs. (6a) and (6b), respectively, by proper exchanging coordinates and parameters. Note that the apostrophes labeling the $\delta$ 's at the right hand sides of Eqs. (6a) and (6b) refer only to the form of indexing the directions but not to the values of the angle changes, since these are invariant against coordinate rotations. The other off-diagonal shear element $\varepsilon_{12}^{\prime}$ can only be determined by examining angle changes for directions out of the planes perpendicular to base vectors $\mathbf{a}, \mathbf{b}$, for instance for $\langle 111\rangle^{\prime}$ type directions in the orthorhombic system.

Recommendations for appropriate choices of directions in different cubic lattice based layer types are listed in Table I together with the corresponding relations between angle changes and component of the deviatory part of the strain tensor. The main criterion for these choices is to minimize the error in the strain tensor components to be deduced: on one hand, the channeling direction should be of low index type; on the other, the angle $\theta$ to the pole direction should be small as can be seen from Eq. (4a). According to columns 2 to 5/last five lines of Table I, at least two independent angle changes are required to determine one strain component. The factors in front of the pairs of angle changes are close to 1 for the main components and somewhat lower (between $1 / 2$ and 3/4) for the nondiagonal components $\varepsilon_{13}^{\prime}$ and $\varepsilon_{23}^{\prime}$. For an accuracy of about $0.042^{\circ}$ in determining changes in angles

TABLE I. Layer type, symmetry, appropriate orthorhombic lattice vectors, a, b, c, direction vectors in the orthorhombic base planes, (010)' and (100)', $[101]^{\prime},[\overline{1} 01]$, and $[011]^{\prime},[0 \overline{1} 1]^{\prime}$, and out of that planes, [hkl] $]^{\prime},[\overline{\mathrm{hkl}}]$, suited to relate the strain tensor components in the orthorhombic coordinate system, $\varepsilon_{\mathrm{ij}}^{\prime}$, to changes in the angle between the pole direction and other directions [hkl], $\delta_{\mathrm{hkl}}$; the angle changes are indexed according to the underlying cubic crystals

\begin{tabular}{|c|c|c|c|c|}
\hline $\begin{array}{l}\text { Layer type } \\
\text { Symmetry }\end{array}$ & $\begin{array}{c}(001) \\
\text { Tetragonal }\end{array}$ & $\begin{array}{c}\text { [110]-S on (001) } \\
\text { Orthorhombic }\end{array}$ & $\begin{array}{l}(011) \\
\text { Orthorhombic }\end{array}$ & $\begin{array}{c}(111) \\
\text { Trigonal/monoclinic }\end{array}$ \\
\hline $\begin{array}{l}\text { Base vectors, } \mathbf{a}, \mathbf{b} \\
\text { pole vector } \mathbf{c}\end{array}$ & {$[100],[010],[001]$} & {$[110],[\overline{1} 10],[001]$} & {$[100],[01 \overline{1}],[011]$} & {$[1 \overline{1} 0],[11 \overline{2}],[111]$} \\
\hline$a, b, c$ & $1,1,1$ & $\sqrt{ } 2, \sqrt{ } 2,1$ & $1, \sqrt{ } 2, \sqrt{ } 2$ & $\sqrt{2}, \sqrt{ } 6, \sqrt{ } 3$ \\
\hline$[101]^{\prime},[\overline{1} 01]^{\prime}$ & {$[101],[\overline{101}]$} & {$[111],[\overline{1} \overline{1} 1]$} & {$[111],[\overline{1} 11]$} & {$[201],[021]$} \\
\hline$[011]^{\prime},[0 \overline{1} 1]^{\prime}$ & {$[011],[0 \overline{1} 1]$} & {$[\overline{1} 11],[1 \overline{1} 1]$} & {$[010],[001]$} & {$[22 \overline{1}],[001]$} \\
\hline$[\mathrm{hkl}]^{\prime},[\overline{\mathrm{hk}} \mathrm{kl}]^{\prime}$ & {$[111],[\overline{1} 11]$} & {$[011],[\overline{1} 01]$} & {$[110],[\overline{1} 10]$} & {$[100],[010]$} \\
\hline $\begin{array}{l}\varepsilon_{11}^{\prime}-\varepsilon_{33}^{\prime} \\
\varepsilon_{13}^{\prime}(=0 ?) \\
\varepsilon_{22}^{\prime}-\varepsilon_{33}^{\prime} \\
\varepsilon_{23}^{\prime}(=0 ?) \\
g_{\prime}^{\prime}\end{array}$ & $\begin{array}{c}\delta_{101}+\delta_{101}^{-} \\
\left(\delta_{101}^{-}-\delta_{101}\right) / 2 \\
\delta_{011}+\delta_{011} \\
\left(\delta_{011}^{-}-\delta_{011}\right) / 2\end{array}$ & $\begin{array}{c}(3 / \sqrt{8})\left(\delta_{111}+\delta_{111}^{--}\right) \\
(3 / 8)\left(\delta_{111}^{-}-\delta_{111}\right) \\
(3 / \sqrt{8})\left(\delta_{111}^{-}+\delta_{111}\right) \\
(3 / 8)\left(\delta_{111}^{-}-\delta_{111}^{-}\right)\end{array}$ & $\begin{array}{c}(3 / \sqrt{8})\left(\delta_{111}+\delta_{111}^{-}\right) \\
(3 / 4)\left(\delta_{111}^{-}-\delta_{111}\right) \\
\delta_{010}+\delta_{001} \\
\left(\delta_{001}-\delta_{010}\right) / 2\end{array}$ & $\begin{array}{c}(5 / \sqrt{24})\left(\delta_{201}+\delta_{021}\right) \\
(5 / 8)\left(\delta_{021}-\delta_{201}\right) \\
(3 / \sqrt{8})\left(\delta_{221}+\delta_{001}\right) \\
(3 / 8)\left(\delta_{001}-\delta_{221}\right)\end{array}$ \\
\hline$\varepsilon_{12}^{\prime}$ & $(3 / \sqrt{8})\left(\delta_{111}-\delta_{111}^{-}\right)$ & $\delta_{011}-\delta_{101}^{-}$ & $\sqrt{3 / 2}\left(\delta_{110}-\delta_{110}^{-}\right)$ & $\sqrt{3 / 2}\left(\delta_{100}-\delta_{010}\right)$ \\
\hline
\end{tabular}
considered here. Since, for angles between directions, the reference direction is always the pole, this need not be included in the labeling of the angle change. Note that the relations for $\varepsilon_{12}^{\prime}$ are restricted to $\varepsilon_{13}^{\prime}=\varepsilon_{13}^{\prime}=0$. For the more general case, angle changes for the directions following from reflection at the $(010)^{\prime}$-plane are needed in addition (angles in radians). 
between two directions, this implies uncertainties in determining certain strain component of about $0.1 \%$ strain for the main components and somewhat smaller uncertainties for $\varepsilon_{13}^{\prime}$ and $\varepsilon_{23}^{\prime}$.

The simplest way to derive the two shear components of the strain tensor parallel to the layer plane, represented by $\varepsilon_{12}^{\prime}$ and $\varepsilon_{11}^{\prime}-\varepsilon_{22}^{\prime}$, is to apply Eqs. (4a) and (4b) to the two orthogonal base planes $(010)^{\prime},(\overline{100})^{\prime}$ and $(010),(\overline{100})$, and to two pairs of nonorthogonal (channeling) planes perpendicular to the layer plane, respectively. Results for different layer types are compiled in Table II. The accuracy in determining strain induced changes of angles between two channeling planes is, however, significantly lower than for changes of angles between directions, say by a factor of 2, which would correspond to an increase in the uncertainty of strain components from $0.1 \%$ to $0.2 \%$.

\section{B. Boundary conditions for perfect surfaces}

The assumption of a force free layer surface implies vanishing of the two shear stress components along the surface and the stress components normal to it (see, for instance, Ref. 18). For perfect surfaces with a crystallographic orientation identical to that of the layer system, this means that, in our nomenclature, $\sigma_{13}^{\prime}\left(\propto \varepsilon_{13}^{\prime}\right), \sigma_{23}^{\prime}\left(\propto \varepsilon_{23}^{\prime}\right)$, and $\sigma_{33}^{\prime}$ $\left(\propto \varepsilon_{11}^{\prime}, \varepsilon_{22}^{\prime}\right)$, are zero. According to Table I, the conditions $\varepsilon_{13}^{\prime}=\varepsilon_{23}^{\prime}=0$ imply the equality of the symmetric angle changes involved, simplifying the associated relations for the diagonal elements $\varepsilon_{11,22}^{\prime}-\varepsilon_{33}^{\prime}$ of the strain tensor. Consequently, if the crystallographic orientation of the surface of the layer system is well established, only one angular channeling yield scan is needed for determining one of each of the diagonal elements $\varepsilon_{11,22}^{\prime}-\varepsilon_{33}^{\prime}$, for instance $\delta_{101}$ and $\delta_{101}$ for (001)-layers or $\delta_{021}$ and $\delta_{001}$ for (111)-layers, respectively. Effects of systematic (long range or local) deviations of the surface orientations from the layer orientation on the strain components are discussed below.

For a layer system with a surface close to the nominal crystallographic orientation, the information required to complete the deviatory part of the strain tensor by a sixth component is provided by the condition of vanishing normal stress, $\sigma_{33}^{\prime}=0$. In the framework of the elastic continuum approach (which has been shown to be applicable even down to SiGe layers consisting only of a few atomic monolayers ${ }^{1}$ ) this yields a Poisson type relation between the normal and parallel strain components $\varepsilon_{33}^{\prime}$ and $\varepsilon_{11}^{\prime}, \varepsilon_{22}^{\prime}$

$$
\varepsilon_{33}^{\prime}=-\left(\rho_{1} \varepsilon_{11}^{\prime}+\rho_{2} \varepsilon_{22}^{\prime}\right)
$$

with $\rho_{1}=C_{13}^{\prime} / C_{33}^{\prime}$ and $\rho_{2}=C_{23}^{\prime} / C_{33}^{\prime}$.

In Eq. (7b), $C_{13}^{\prime}, C_{23}^{\prime}$, and $C_{33}^{\prime}$ are the appropriate elastic constants, in the Voigt notation, of the layer system considered. For cubic base symmetry assumed in the present paper, the elastic constant matrix may be expressed by the Voigt elastic constants, $C_{11}, C_{12}, C_{44}$, as

$$
\begin{aligned}
C_{i j k l}= & C_{12} \delta_{i j} \delta_{k l}+C_{44}\left(\delta_{i k} \delta_{j l}+\delta_{i l} \delta_{j k}\right) \\
& +\left(C_{11}-C_{12}-2 C_{44}\right) \delta_{i j k l},
\end{aligned}
$$

where the twice indexed quantity, $\delta_{i j}=1$ for $i=j, \delta_{i j}=0$ for $i \neq j$, is the common Kronecker-delta, and $\delta_{i j k l}=1$ for $i=j$ $=k=l$ and $\delta_{i j k l}=0$, otherwise. Note, that $C_{11}-C_{12}-2 C_{44}=0$ for elastic isotropy.

The elastic constant matrix of a orthorhombic layer is obtained from that of the cubic base described by Eq. (8) by applying the transformation matrix formed by the three unit vectors in the orthorhombic coordinate directions as given in Table I (for such transformations see also Ref. 18). The result of this procedure may be expressed in the form

$$
\begin{gathered}
C^{\prime}=C+\left(C_{11}-C_{12}-2 C_{44}\right) S(\mathbf{u}, \mathbf{v}) \\
\text { with } S=\sum_{i} u_{i}^{2} v_{i}^{2} /(u v)^{2}
\end{gathered}
$$

The left hand side of Eq. (9), $C^{\prime}$, represents the orthorhombic elastic constants, the right hand side the pertinent linear combination of the associated cubic elastic constants, in which each factor $S(\mathbf{u}, \mathbf{v})$ contains the squares of two properly selected members out of the three orthorhombic lattice vectors. In detail, $C=C_{12}+2 C_{44}, \quad$ and $\mathbf{u}=\mathbf{v}=\mathbf{a}, \mathbf{b}, \mathbf{c}$ for $C_{11}^{\prime}, C_{22}^{\prime}, C_{33}^{\prime} ; C=C_{12}$, and $(\mathbf{u}, \mathbf{v})=(\mathbf{a}, \mathbf{b}),(\mathbf{a}, \mathbf{c}),(\mathbf{b}, \mathbf{c})$ for $C_{12}^{\prime}, C_{13}^{\prime}, C_{23}^{\prime} ; C=C_{44}$, and $(\mathbf{u}, \mathbf{v})=(\mathbf{b}, \mathbf{c}),(\mathbf{a}, \mathbf{c}),(\mathbf{a}, \mathbf{b})$, for $C_{44}^{\prime}, C_{55}^{\prime}, C_{66}^{\prime}$, respectively. To give an example, we consider $C_{66}^{\prime}$ for the (111) layer defined in column 5 of Table I: with the lattice vectors $\mathbf{a}=(1 \overline{10})$ and $\mathbf{b}=(11 \overline{2}), S(\mathbf{a}, \mathbf{b})=(1$ $+1+0) / 12=1 / 6$ resulting in $C_{66}^{\prime}=\left(C_{11}-C_{12}+4 C_{44}\right) / 6$.

We are here first of all interested in the two components of the biaxial Poisson ratio, $\rho_{1}$ and $\rho_{2}$. For layer systems with

TABLE II. Layer type, orthorhombic base lattice constants, $a, b$, orthorhombic base and appropriate nonbase planes, $(010)^{\prime},(\overline{100})^{\prime}$, and $(\overline{1} 10)^{\prime}$, suited to determine the strain tensor components in the orthorhombic base system, $\varepsilon_{12}^{\prime}$ and $\varepsilon_{11}^{\prime}-\varepsilon_{22}^{\prime}$, respectively, as a function of changes in the angles between that planes, which are indexed according to the underlying cubic crystals considered here. Since there is no distinct reference plane, the indices of both planes must be included in denoting the angles between planes. Note that the sequence of the planes $(010)^{\prime},(\overline{1} 10)^{\prime},(\overline{100})^{\prime}$ and the angles between the first and the two following are chosen to be counter clockwise.

\begin{tabular}{lccc}
\hline \hline Layer type & $(001)$ & $(011)$ & $(111)$ \\
\hline$a, b$ & 1,1 & $1, \sqrt{2}$ & $\sqrt{2}, \sqrt{6}$ \\
$(010)^{\prime},(\overline{1} 00)^{\prime}$ & $(010),(\overline{1} 00)$ & $(01 \overline{1}),(\overline{1} 00)$ & $(11 \overline{2}),(\overline{1} 10)$ \\
$(\overline{1} 10)^{\prime}$ & $(\overline{1} 10)$ & $(\overline{1} 1 \overline{1})$ & $(01 \overline{1})$ \\
$2 \varepsilon_{12}^{\prime}$ & $\delta_{010, \overline{1} 00}$ & $\delta_{01 \overline{1}, \overline{1} 0}$ & $\delta_{11 \overline{2}, \overline{1} 10}$ \\
$\varepsilon_{11}^{\prime}-\varepsilon_{22}^{\prime}$ & $2 \delta_{010, \overline{1} 10}-2 \varepsilon_{12}$ & $(3 / \sqrt{2}) \delta_{01 \overline{1}, \overline{1} 1 \overline{1}}-\sqrt{2} \varepsilon_{12}$ & $(4 / \sqrt{3}) \delta_{11 \overline{2}, 01 \overline{1}}-\varepsilon_{12} / \sqrt{3}$ \\
\hline \hline
\end{tabular}


high rotational symmetry above twofold (e.g., tetragonal, trigonal as in columns 2, 3, and 5 in Table I) $S(\mathbf{a , c})$ $=S(\mathbf{b}, \mathbf{c})$ implying $C_{13}^{\prime}=C_{23}^{\prime}$ and, according to Eq. (7b), $\rho_{1}$ $=\rho_{2}$. In less symmetric cases, the two components of the biaxial Poisson ratio must be considered in general unequal, $\rho_{1} \neq \rho_{2}$. For (011) layer systems on a cubic substrate (column 4 in Table I), for instance, $S(\mathbf{a}, \mathbf{c})=0$, and $S(\mathbf{b}, \mathbf{c})=S(\mathbf{c}, \mathbf{c})$ $=1 / 2$ yield $C_{13}^{\prime}=C_{12}, C_{23}^{\prime}=\left(C_{11}+C_{12}-2 C_{44}\right) / 2, C_{33}^{\prime}=\left(C_{11}\right.$ $\left.+C_{12}+2 C_{44}\right) / 2$ and consequently $\rho_{1}=2 C_{12} /\left(C_{11}+C_{12}\right.$ $\left.+2 C_{44}\right)$ and $\rho_{2}=\left(C_{11}+C_{12}-2 C_{44}\right) /\left(C_{11}+C_{12}+2 C_{44}\right)$.

Abbreviating the relations between the diagonal elements of the deviatory strain tensor and the corresponding angle changes given in Table I as $\varepsilon_{11,22}^{\prime}-\varepsilon_{33}^{\prime} \equiv e_{1,2}$ and using Eq. (6a) for eliminating $\varepsilon_{33}^{\prime}$ we may write $\varepsilon_{11,22}^{\prime}$ as

$$
\begin{aligned}
& \varepsilon_{11}^{\prime}=\left[\left(1+\rho_{2}\right) e_{1}-\rho_{2} e_{2}\right] /\left(1+\rho_{1}+\rho_{2}\right), \\
& \varepsilon_{22}^{\prime}=\left[\left(1+\rho_{1}\right) e_{2}-\rho_{1} e_{1}\right] /\left(1+\rho_{1}+\rho_{2}\right),
\end{aligned}
$$

\section{Boundary conditions for misoriented surfaces}

So far, we have assumed that the crystallographic orientations of the layer system and the surface are identical. This is, however, not necessarily realized in practical cases. Conceivable are large scale misorientations (over the millimeter scale of the He bombarded area) and medium-scale misorientations (over scales small compared to the bombarded area but large compared to the thickness of the layer; for instance asymmetric saw tooth shaped misorientations formed as a result of directed cutting or grinding). Such deviations in the surface from the ideal case may be undesired or intentional (for instance to support crystal growth by regular arrays of surface steps).

Any type of misorientation of the surface must be considered to affect the nominal boundary conditions, i.e., to induce finite values of the involved strain components, $\varepsilon_{13}^{\prime}$, $\varepsilon_{23}^{\prime}$, and $\varepsilon_{33}^{\prime}$ (note that $\varepsilon_{13}^{\prime}$ and $\varepsilon_{23}^{\prime}$ do not represent direct measures of the surface inclination). In the following, we derive relations between these strain components and the tilt of surface of the orthorhombic layer, the magnitude and orientation of which we describe by a rotation of the original to a surface adapted coordinate system (denoted by *) according to Euler angles for "nutation" (tilting), "precession" (direction of tilting), and "intrinsic rotation," $\theta, \psi$, and $\varphi$, respectively, choosing $\varphi=-\psi$ to guarantee the formal $x-y$ exchange symmetry. For expected small tilt angle, $\theta \ll 1$, the corresponding rotation matrix, $\mathbf{M}(\theta, \psi)$ may linearized with respect $\theta$ resulting in

$$
\mathbf{M}(\theta, \psi) \approx\left(\begin{array}{ccc}
1 & 0 & \alpha_{1} \\
0 & 1 & \alpha_{2} \\
-\alpha_{1} & -\alpha_{2} & 1
\end{array}\right)
$$

where $\alpha_{1}=-\theta \sin \psi$ and $\alpha_{2}=\theta \cos \psi$ have been introduced as the two components of the surface gradient. Applying this approximate rotation matrix to the stress tensor in the original coordinate system of the orthorhombic layer and selecting the relevant stress components vanishing at the inclined surface, $\sigma_{13}^{*}=\sigma_{23}^{*}=\sigma_{33}^{*}=0$, we find the relations

$$
\begin{aligned}
& \sigma_{13} \approx \alpha_{1}\left(\sigma_{11}-\sigma_{33}\right)+\alpha_{2} \sigma_{12} \approx \alpha_{1} \sigma_{11}+\alpha_{2} \sigma_{12}, \\
& \sigma_{23} \approx \alpha_{2}\left(\sigma_{22}-\sigma_{33}\right)+\alpha_{1} \sigma_{12} \approx \alpha_{2} \sigma_{22}+\alpha_{1} \sigma_{12}, \\
& \sigma_{33} \approx 2 \alpha_{1} \sigma_{13}+2 \alpha_{2} \sigma_{23}=0\left(\alpha_{1}^{2}, \alpha_{2}^{2}\right) .
\end{aligned}
$$

Using $\sigma_{13}$ and $\sigma_{23}$ as given by Eqs. (12a) and (12b) in Eq. (12c) we see that the misorientation induced change in the boundary condition for stress components normal to the nominal surface, $\sigma_{33}$, is of second order in $\alpha_{1,2}$ and has, therefore, to be neglected consistently within our linear approximation. An important consequence of this is that $\sigma_{33}$ $\approx 0$ and, with this, Eqs. (7) and (10) remain very good approximations for moderately misoriented surfaces.

In the context of strain measurements by RBS/C discussed in this paper, it is useful to relate in Eqs. (12a) and (12b) the stress to the strain components. It is sufficient to consider Eq. (12a) for $\alpha_{2}=0$ resulting in the relation

$$
\begin{aligned}
\varepsilon_{13} & \approx \alpha_{1}\left[\left(C_{11}^{\prime}-C_{13}^{\prime}\right) \varepsilon_{11}+\left(C_{12}^{\prime}-C_{23}^{\prime}\right) \varepsilon_{22}\right] / C_{55}^{\prime} \approx 2 \alpha_{1} \varepsilon_{11} \\
& \approx 2 \alpha_{1} \varepsilon_{11} \text { for elastic isotropy. }
\end{aligned}
$$

An analogous relation holds for $\varepsilon_{23}$.

According to Eq. (13), the value of $\varepsilon_{13}$ is below the resolution limit of RBS/C of about $0.05 \%$ for $\varepsilon_{11,22}$ of about $1 \%$ and $\alpha_{1,2}<2.5 \%$ (in radians) corresponding to an inclination of about $1.5^{\circ}$. An important conclusion of this is that the shear stress components $\varepsilon_{13,23}$ can be safely neglected if some information guarantees that large or medium scale misorientations are below the commonly assumed upper limit of about $1^{\circ}$.

\section{Orthorhombic strain tensors}

An important limiting case is realized when RBS/C measurements have established that the in-plane shear stress $\varepsilon_{12}^{\prime}$ vanishes (or is negligible), showing that the symmetry of the strain tensor corresponds to the intrinsic symmetry of the layer system. In this case, the diagonal elements define the eigenvalues of the strain tensor, $\boldsymbol{\varepsilon}_{\mathbf{1}}=\varepsilon_{11}^{\prime}, \boldsymbol{\varepsilon}_{\mathbf{2}}=\varepsilon_{22}^{\prime}$. These are equal for epitaxial (pseudomorphic) layers but must be considered to be different for asymmetrically relaxed layers. Asymmetrical layer relaxation may occur by asymmetrical (biased) activation of dislocation slip systems in layers of reduced symmetry, for instance by a restriction of the glide of dislocations with $\langle 110\rangle / 2$ Burgers vectors to (111) and (111) planes in (011)-layers. According to Eqs. (10a) and (10b), the degrees of relaxation in the tangential directions $1^{\prime}$ and $2^{\prime}$ of a layer may be written as:

$$
\begin{aligned}
R_{1,2} & =1-\varepsilon_{1,2} / \varepsilon_{0} \\
& =1-\left[\left(1+\rho_{2,1}\right) e_{1,2}-\rho_{2,1} e_{2,1}\right] /\left[\varepsilon_{0}\left(1+\rho_{1}+\rho_{2}\right)\right],
\end{aligned}
$$

where $\varepsilon_{0}$ is the extreme value of the strain defined by perfect epitaxial connection between the layers and the substrate $\left(\varepsilon_{0}=-0.0417 x\right.$ in the case of $\left.\mathrm{Si}_{1-x} \mathrm{Ge}_{x} \varepsilon_{0}\right)$. In strain engineering of nanoelectronic devices, the asymmetric relaxation degrees $R_{1,2}$ are the key quantities to be determined by RBS/C. 


\section{E. Application to strain relaxation in $\{110\}$ oriented SiGe layers}

As mentioned in the introduction, asymmetric strain relaxation in SiGe layers on $\mathrm{Si}$ is desirable for engineering strain states favoring high carrier mobilities. This can only be achieved by reducing the tetragonal symmetry of the commonly used $\{100\} \mathrm{SiGe} / \mathrm{Si}$ layers. Asymmetric strain relaxation in orthorhombic $\{110\} \mathrm{SiGe} / \mathrm{Si}$ layers has been recently investigated by RBS/C using the results of the present study to evaluate the RBS/C data. ${ }^{16}$

For this specific case, we may use the results given in column 4 of Table I in conjunction with Eq. (10). In Ref. 16, we have tacitly assumed a perfect surface with a nominal orientation equal to the $\{110\}$ orientation of the layer. In this case, $\varepsilon_{13}^{\prime}=\varepsilon_{23}^{\prime}=0$ meaning $\delta_{111}=\delta_{-111}, \delta_{010}=\delta_{001}$, and, according to Eqs. (10a) and (10b)

$$
\begin{aligned}
& \varepsilon_{11}^{\prime} \approx\left[(3 / \sqrt{ } 2)\left(1+\rho_{2}\right) \delta_{111}-2 \rho_{2} \delta_{010}\right] /\left(1+\rho_{1}+\rho_{2}\right), \\
& \varepsilon_{22}^{\prime} \approx\left[2\left(1+\rho_{1}\right) \delta_{010}-(3 / \sqrt{ } 2) \rho_{1} \delta_{111}\right] /\left(1+\rho_{1}+\rho_{2}\right),
\end{aligned}
$$

where $\rho_{1}=0.329$ and $\rho_{2}=0.181$ for (011)SiGe layers. According to Table I and II, the in-plane nondiagonal element $\varepsilon_{12}^{\prime}$ is given in terms of angle changes between directions and planes, respectively, as

$$
\begin{aligned}
& \varepsilon_{12}^{\prime}(\text { directions }) \approx \sqrt{ }(3 / 2)\left(\delta_{110}-\delta_{-110}\right), \\
& \left.\varepsilon_{12}^{\prime} \text { (planes }\right) \approx \delta_{01 \overline{1}, \overline{1} 00} / 2
\end{aligned}
$$

When RBS/C measurements yield, within the experimental accuracy, $\varepsilon_{12}^{\prime} \approx 0$, the strain tensor may be considered to have the orthorhombic symmetry of the layer, with generally two different eigenvalues $\boldsymbol{\varepsilon}_{\mathbf{1 , 2}}=\varepsilon_{11,22}^{\prime}$, which can be used to define two different degrees of relaxation $R_{1,2}=1-\varepsilon_{1,2} / \varepsilon_{0}$ where $\varepsilon_{0}=-0.0417 x$ for $\mathrm{Si}_{1-x} \mathrm{Ge}_{x}$.

In the following, we briefly report on the evaluation of data sets for RBS/C measurements on $50 \mathrm{~nm}$ thick (011) $\mathrm{Si}_{0.833} \mathrm{Ge}_{0.167} / \mathrm{Si}$ samples $\left(\varepsilon_{0}=0.70 \%\right)$ before and after relaxation by $\mathrm{He}$ implantation and subsequent annealing. For the as grown sample, $\delta_{111}=-0.26^{\circ} \pm 0.04^{\circ}, \quad \delta_{010}$ $=-0.25^{\circ} \pm 0.04^{\circ}$, resulting with Eqs. (15a) and (15b) in $\varepsilon_{11}^{\prime}$ $=\varepsilon_{1}=(0.70 \pm 0.16) \%, \quad \varepsilon_{22}^{\prime}=\varepsilon_{2}=(0.63 \pm 0.15) \%$ correspond ing to $R_{1}=(0 \pm 0.2), R_{2}=(0.10 \pm 0.2)$, respectively. Thus, the as grown layer system is almost pseudomorphic. Implantation of $10 \mathrm{keV} \mathrm{He}$ ions to a dose of $7 \times 10^{15} / \mathrm{cm}^{2}$ and subsequent annealing changes the data set to $\delta_{111}=$ $-0.09^{\circ} \pm 0.04^{\circ}$ $\delta_{010}$ $=-0.246^{\circ} \pm 0.04^{\circ}, \quad \varepsilon_{1}=(0.25 \pm 0.16) \%, \quad \varepsilon_{2}=(0.63 \pm 0.15) \%$ corresponding to $R_{1}=0.64 \pm 0.2, R_{2}=0.10 \pm 0.2$. This change shows that the treatment has induced a purely uniaxial relaxation of the (011)SiGe layer in the [100]-direction which may be interpreted as a restriction of the glide of dislocations with $\langle 110\rangle / 2$ Burgers vectors on (111) and (111) planes to the $[01 \overline{1}]$-direction. ${ }^{16}$ The errors given are with the reservation that the examined layers are fully orthorhombic including the surface, i.e., that $\varepsilon_{12}^{\prime}=0$ as well as $\varepsilon_{13}^{\prime}=\varepsilon_{23}^{\prime}=0$. Ac- cording to Sec. II C, the latter condition can be considered to be fulfilled within the experimental accuracy if the medium and large scale surface misorientations are shown to be below $2.5^{\circ}$.

We can check the latter assumption by examining five independent direction changes necessary to deduce the complete deviatory part of the strain tensor. The highest accuracy would be obtained by choosing the directions recommended in Table I. This would require, however, a rotation of the sample in the RBS equipment by $180^{\circ}$ to obtain two of the recommended five direction changes. To avoid this, we have examined the changes in the angles between the pole direction [011] and the directions [010], [110], [111], [101], and [001]. By solving the proper set of equations of the type of Eq. (5), the five required strain components can be deduced from the five measured angle changes. We give, as an example, the results for the strain components (in percent) for an as grownSi $\mathrm{Si}_{0.838} \mathrm{Ge}_{0.162} / \mathrm{Si}$ sample (with $\varepsilon_{0}=-0.68 \%$ )

$$
\begin{aligned}
& \varepsilon_{11}^{\prime}=-(0.70 \pm 0.26) \%, \quad \varepsilon_{22}^{\prime}=-(0.50 \pm 0.15) \%, \\
& \varepsilon_{12}^{\prime}=-(0.014 \pm 0.17) \%, \\
& \varepsilon_{13}^{\prime}=-(0.10 \pm 0.10) \%, \quad \varepsilon_{23}^{\prime}=-(0.0026 \pm 0.06) \% .
\end{aligned}
$$

Within the given errors (again based on an error of $\pm 0.03^{\circ}$ in determining a certain direction) the nondiagonal components of the strain tensor $\varepsilon_{12}^{\prime}$ and $\varepsilon_{23}^{\prime}$ are clearly zero; only for $\varepsilon_{13}^{\prime}$, a finite value cannot be fully ruled out which would indicate some medium or large scale surface gradient in [100] direction (note that our convenience of avoiding large angle sample rotations results in an increased error in $\varepsilon_{11}^{\prime}$ since, for this, five angle measurements are required). The uncertainty in $\varepsilon_{13}^{\prime}$ underlines the importance of using information on a possible (local) surface misorientation in conjunction with our analysis of the relation between this and the nondiagonal components of the strain tensor $\varepsilon_{13}^{\prime}$ and $\varepsilon_{23}^{\prime}$.

\section{CONCLUSIONS}

We have presented a systematic derivation of relations between changes in angles between channeling crystal directions and components of the strain tensor for general crystalline layer systems of reduced symmetry compared to the basic (cubic) crystal. The simplest method to derive such relations, applicable to practically all cases of interest, is to describe the layer systems as appropriately chosen pseudoorthorhombic systems. For important layer types, choices of crystal directions most suited to minimize the error in the deduced strain tensor are suggested. We have discussed the effects of possible medium or large scale surface misorientations on the commonly assumed boundary conditions and the associated strain tensor components. A possible asymmetry in the principal axes components of the strain tensor for layers of low symmetry is attributed to asymmetric strain relaxation resulting from a restriction in the slip system of the dislocations inducing the relaxation. Our results are considered to support the use of RBS/C for controlling and characterizing advanced strain engineering in $\mathrm{Si} / \mathrm{SiGe}$ layer sys- 
tems. To illustrate the method we have applied the results to strain relaxation in $\{110\}$ oriented SiGe layers.

\section{ACKNOWLEDGMENTS}

This work was partially supported by the German Federal Ministry of Education and Research and the French Ministry of Industry via the MEDEA ${ }^{+}$project DECISIF (2T 104) and by the European Community's Seventh Framework Programme (Grant No. FP7/2007-2013) under Grant Agreement No. 216171.

${ }^{1}$ L. C. Feldman, J. Bevk, B. A. Davidson, H.-J. Gossmann, and J. P. Mannaerts, Phys. Rev. Lett. 59, 664 (1987).

${ }^{2}$ M. L. Lee, E. A. Fitzgerald, M. T. Bulsara, M. T. Currie, and A. Lochtefeld, J. Appl. Phys. 97, 011101 (2005).

${ }^{3}$ H. Trinkaus, B. Holländer, S. Rongen, S. Mantl, H.-J. Herzog, J. Kuchenbecker, and T. Hackbarth, Appl. Phys. Lett. 76, 3552 (2000).

${ }^{4}$ T. Tezuka, N. Hirashita, Y. Moriyama, S. Nakaharai, N. Sugiyama, and S. Takagi, Appl. Phys. Lett. 90, 181918 (2007).

${ }^{5}$ D. Buca, B. Holländer, H. Trinkaus, S. Mantl, R. Carius, R. Loo, M. Caymax, and H. Schaefer, Appl. Phys. Lett. 85, 2499 (2004).
${ }^{6}$ Y. Sun, S. E. Thompson, and T. Nishida, J. Appl. Phys. 101, 104503 (2007).

N. S. Bennett, N. E. B. Cowern, A. J. Smith, R. M. Gwilliam, B. J. Sealy, L. O'Reilly, P. J. McNally, G. Cooke, and H. Kheyrandish, Appl. Phys. Lett. 89, 182122 (2006).

${ }^{8}$ G. A. Rozgonyi and D. C. Miller, Thin Solid Films 31, 185 (1976).

${ }^{9}$ E. Kasper and H. J. Herzog, Thin Solid Films 44, 357 (1977).

${ }^{10}$ K. Z. Troost, P. van der Sluis, and D. J. Gravesteijn, Appl. Phys. Lett. 62, 1110 (1993).

${ }^{11}$ J. C. Bean, L. C. Feldman, A. T. Fiory, S. Nakahara, and I. K. Robinson, J. Vac. Sci. Technol. A 2, 436 (1984).

${ }^{12}$ S. T. Picraux, L. R. Dawson, J. Y. Tsao, B. L. Doyle, and S. R. Lee, Nucl. Instrum. Methods Phys. Res. B 33, 891 (1988).

${ }^{13}$ H.-J. Gossmann and J. P. Mannaerts, Phys. Rev. B 39, 10108 (1989).

${ }^{14}$ B. Holländer, H. Heer, M. Wagener, H. Halling, and S. Mantl, Nucl. Instrum. Methods Phys. Res. B 161-163, 227 (2000).

${ }^{15}$ D. Buca, B. Holländer, S. Feste, St. Lenk, H. Trinkaus, S. Mantl, R. Loo, and M. Caymax, Appl. Phys. Lett. 90, 032108 (2007).

${ }^{16}$ R. A. Minamisawa, D. Buca, H. Trinkaus, B. Holländer, S. Mantl, V. Destefanis, and J. M. Hartmann, Appl. Phys. Lett. 95, 034102 (2009).

${ }^{17}$ B. R. Appleton and G. Foti, Ion Beam Handbook for Material Analysis (Academic, New York, 1977).

${ }^{18}$ M. Murakami and T.-S. Kuan, Treatise on Materials Science and Technology (Academic Press, New York, 1982), Vol. 24. 